\title{
On Riemann problem in weighted Smirnov classes with general weight
}

\author{
Bilal Bilalov, Aysel Guliyeva, and Sabina Sadigova
}

\begin{abstract}
Weighted Smirnov classes in bounded and unbounded domains are defined in this work. Nonhomogeneous Riemann problems with a measurable coefficient whose argument is a piecewise continuous function are considered in these classes. A Muckenhoupt type condition is imposed on the weight function and the orthogonality condition is found for the solvability of nonhomogeneous problem in weighted Smirnov classes, and the formula for the index of the problem is derived. Some special cases with power type weight function are also considered, and conditions on degeneration order are found.
\end{abstract}

\section{Introduction}

Let $\Gamma$ be some rectifiable Jordan contour on the complex plane $\mathbb{C}$ and let $X(\Gamma)$ be a Banach space of functions on $\Gamma$. Let $E_{X}^{+}(\operatorname{int} \Gamma)$ and $E_{X}^{-}(\operatorname{ext} \Gamma)$ be the Smirnov classes of functions analytic in int $\Gamma$ and ext $\Gamma$, respectively, generated by the metric of $X(\Gamma)$. Let $G: \Gamma \rightarrow \mathbb{C}$ and $g \in X(\Gamma)$ be some given functions. Consider the Riemann problem

$$
\begin{aligned}
& F^{+}(\tau)-G(\tau) F^{-}(\tau)=g(\tau), \tau \in \Gamma, \\
& \left(F^{+} ; F^{-}\right) \in E_{X}^{+}(\operatorname{int} \Gamma) \times E_{X}^{-}(\operatorname{ext} \Gamma),
\end{aligned}
$$

where $F^{+}(\tau)\left(F^{-}(\tau)\right)$ means the nontangential boundary value of the function $F^{+} \in E_{X}^{+}(\operatorname{int} \Gamma)\left(F^{-} \in E_{X}^{-}(\operatorname{ext} \Gamma)\right)$ at a point $\tau \in \Gamma$ from the inside $\Gamma$ (from the outside $\Gamma$ ). Here $G(\cdot)$ is called the coefficient, and $g(\cdot)$ the right-hand side of problem (1). This problem has a deep history and is well studied in different formulations. Monographs of various mathematicians are devoted to this direction (see, for example, $[10,13,15,20,28,29,30]$ ). $L_{p}$-theory was developed in the monograph [10]. The case of a weighted

Received July 30, 2020.

2020 Mathematics Subject Classification. 30E25, 32A55.

Key words and phrases. Weighted Smirnov classes, Riemann problem, Muckenhoupt condition.

https://doi.org/10.12697/ACUTM.2021.25.03 
Lebesgue space with power weight was considered in [16, 17, 23, 24, 25, 27]. Namely, the general solution of the homogeneous Riemann problem with a piecewise-continuous coefficient in the Smirnov weight classes with a weight of the general form is constructed in the papers [23, 24, 27]. In [27], a general solution to the nonhomogeneous Riemann problem with a piecewise continuous coefficient in the weighted Smirnov classes with the weight of a power form is found. In paper [25], nonhomogeneous Riemann problem with a piecewise Hölder coefficient is considered, and a particular solution to this problem is found in Smirnov weight classes with a general weight. The Riemann problems associated with the metrics of the Lebesgue space with a variable exponent of summability and Morrey spaces were studied in $[7,8,14,21]$. In [1]-[6], the Riemann problem is used to study the basic properties of special systems of functions in Lebesgue spaces. It should be noted that in all these works, strong restrictions are imposed on jumps of the argument of the coefficient of the problem and on the weight function.

Weighted Smirnov classes in bounded and unbounded domains are defined in this work. Nonhomogeneous Riemann problems with a measurable coefficient whose argument is a piecewise continuous function are considered in these classes. A Muckenhoupt type condition is imposed on the weight function and the orthogonality condition is found for the solvability of nonhomogeneous problem in weighted Smirnov classes, and the formula for the index of the problem is derived. Some special cases with power type weight function are also considered, and conditions on degeneration order are found.

It should be noted that problems close to the topic of this article are considered in works $[31,32]$ and the weighted Hardy classes (we call them weighted Smirnov classes) are defined in a different way and impose more restrictive conditions on the weight function. Analogues of classical results are established in these classes.

\section{Necessary information and auxiliary facts}

In this section, we state some notations and facts to be used to obtain our results. By $O_{r}\left(z_{0}\right)$ we denote a circle of radius $r$ centered at $z_{0}$ on the complex plane $\mathbb{C}$, i.e., $O_{r}\left(z_{0}\right) \equiv\left\{z \in \mathbb{C}:\left|z-z_{0}\right|<r\right\} .|M|$ means the Lebesgue (linear) measure of the set $M \subset \Gamma$, where $\Gamma \subset \mathbb{C}$ is some rectifiable curve. By $\overline{n, m}$ we denote the set $\{n, n+1, \ldots, m\}$, and $[x]$ means the integer part of a number $x \in \mathbb{R}$. Notation $f(x) \sim g(x), x \in M$, means that there exists $\delta>0$ with

$$
0<\delta \leq\left|\frac{f(x)}{g(x)}\right| \leq \delta^{-1}, \quad x \in M
$$

Let us give a definition for Carleson curve. 
Definition 1. A Jordan rectifiable curve $\Gamma$ on the complex plane is called a Carleson curve or a regular curve if

$$
\sup _{z \in \Gamma}\left|\Gamma \cap O_{r}(z)\right| \leq c r, \quad r>0,
$$

where $c$ is a constant independent of $r$.

For more information about this concept see, e.g., [11, 12].

Let $\Gamma$ be some Jordan rectifiable curve and $\omega(\cdot)$ be a weight function on $\Gamma$, i.e., $\omega(\xi)>0$ for a.e. $\xi \in \Gamma$.

Definition 2. We will say that the weight function $\omega: \Gamma \rightarrow \mathbb{R}^{+}=$ $(0,+\infty)$ belongs to the Muckenhoupt class $A_{p}(\Gamma)(p>1)$, if

$$
\sup _{z \in \Gamma} \sup _{r>0}\left(\frac{1}{r} \int_{\Gamma \cap O_{r}(z)} \omega(\xi)|d \xi|\right)\left(\frac{1}{r} \int_{\Gamma \cap O_{r}(z)}|\omega(\xi)|^{-\frac{1}{p-1}}|d \xi|\right)^{p-1}<+\infty .
$$

We will need some facts about the weights $\omega(\cdot)$, which satisfy the Muckenhoupt condition $A_{p}(\Gamma), 1<p<+\infty$, on the rectifiable curve $\Gamma$. The following statement is true.

Statement 1. i) If $\omega \in A_{p}(\Gamma), 1<p<+\infty$, then $\omega \in A_{q}(\Gamma)$ for $q>p$; ii) $\omega \in A_{p}(\Gamma), 1<p<+\infty$, if and only if $\omega^{-\frac{1}{p-1}} \in A_{p^{\prime}}(\Gamma), \frac{1}{p}+\frac{1}{p^{\prime}}=1$; iii) if $\omega \in A_{p}(\Gamma), 1<p<+\infty$, then $\omega \in A_{q}(\Gamma)$ for some $q$ with $1<q<p$.

We will also use the following statement by Coifman and Fefferman [9].

Statement 2. If the function $\omega(\cdot)>0$ satisfies the Muckenhoupt condition $A_{p}(\Gamma), 1<p<+\infty$, then for sufficiently small $\delta>0$ the "inverse Hölder inequality"

$$
\left(\frac{1}{r} \int_{\Gamma \cap O_{r}(z)}|\omega(\xi)|^{1+\delta}|d \xi|\right)^{\frac{1}{1+\delta}} \leq c\left(\frac{1}{r} \int_{\Gamma \cap O_{r}(z)} \omega(\xi)|d \xi|\right), \quad r>0, z \in \Gamma
$$

holds, where $c=c(\delta)$ is a constant independent of $r$ and $z \in \Gamma$.

As usual, by $L_{p, \omega}(\Gamma)$ we denote the weighted Lebesgue space of functions endowed with the norm

$$
\|f\|_{L_{p, \omega}(\Gamma)}=\left(\int_{\Gamma}|f(\xi)|^{p} \omega(\xi)|d \xi|\right)^{\frac{1}{p}} .
$$

Consider the singular Cauchy operator

$$
S_{\Gamma}(f)=\frac{1}{2 \pi i} \int_{\Gamma} \frac{f(\xi)}{\xi-\tau} d \xi, \quad \tau \in \Gamma
$$

The following theorem is valid. 
Theorem D. The operator $S_{\Gamma}$ is bounded in $L_{p}(\Gamma), 1<p<+\infty$, only when $\Gamma$ is a regular curve. Moreover, if $\Gamma$ is a regular curve, then the singular operator $S_{\Gamma}$ is bounded in $L_{p, \omega}(\Gamma), 1<p<+\infty$, only when $\omega \in A_{p}(\Gamma)$.

For more information about these results see, e.g., [11, 12]. We will also use the following result.

Privalov theorem. The following statements hold true: i) the singular operator

$$
S_{\Gamma} f(z)=\frac{1}{2 \pi i} \int_{\Gamma} \frac{f(\xi)}{\xi-z} d \xi, \quad z \in \Gamma,
$$

acts and is bounded in the space Lip $\operatorname{Li}_{\alpha}(\Gamma)$, if $\Gamma$ is a piecewise smooth curve with no cusps; ii) the same statement remains true when $\Gamma$ is a Radon curve with no cusps.

Recall that the Lipschitz space $\operatorname{Lip}_{\alpha}(\Gamma)$ is a Banach space on $\Gamma$ with the norm

$$
\|f\|_{L_{i p_{\alpha}(\Gamma)}}=\max _{z \in \Gamma}|f(z)|+\sup _{z_{1} ; z_{2} \in \Gamma} \frac{\left|f\left(z_{1}\right)-f\left(z_{2}\right)\right|}{\left|z_{1}-z_{2}\right|^{\alpha}} .
$$

For more information about this result see, e.g., [10] and [22].

Let $G(\xi)=|G(\xi)| e^{i \theta(\xi)}$ be complex-valued functions on the curve $\Gamma$. We make the following basic assumptions on the coefficient $G(\cdot)$ of the considered boundary value problem and $\Gamma$ :

(i) $|G(\cdot)|^{ \pm 1} \in L_{\infty}(\Gamma)$;

(ii) $\theta(\cdot)$ is piecewise continuous on $\Gamma$, and $\left\{\xi_{k}: k \in \overline{1, r}\right\} \subset \Gamma$ are discontinuity points of the function $\theta(\cdot)$ :

(iii) $\Gamma$ is either Lyapunov or Radon curve (i.e., it is a limited rotation curve) with no cusps. Direction along $\Gamma$ will be considered as positive, i.e., when moving along this direction the domain D stays on the left side. Let $a \in \Gamma$ be an initial (and also a final) point of the curve $\Gamma$. We will assume that $\xi \in \Gamma$ follows the point $\tau \in \Gamma$, i.e. $\tau \prec \xi$, if $\xi$ follows $\tau$ when moving along a positive direction on $\Gamma \backslash a$, where $a \in \Gamma$ represents two stuck points $a^{+}=a^{-}$, with $a^{+}$a beginning, and $a^{-}$an end of the curve $\Gamma$.

So, without loss of generality, we will assume that $a^{+} \prec \xi_{1} \prec \cdots \prec \xi_{r} \prec$ $b=a^{-}$. Denote one-sided $\operatorname{limits}_{\substack{\xi \rightarrow \xi_{0} \pm 0 \\ \xi \in \Gamma}} g(\xi)$ of the function $g(\xi)$ at the point $\xi_{0} \in \Gamma$ generated by this order by $g\left(\xi_{0} \pm 0\right)$, respectively. The jumps of the function $\theta(\xi)$ at the points $\xi_{k}, k \in \overline{1, r}$, are denoted by $h_{k}: h_{k}=$ $\theta\left(\xi_{k}+0\right)-\theta\left(\xi_{k}-0\right), k \in \overline{1, r}$.

Let $D^{+} \subset \mathbb{C}$ be a bounded domain with the boundary $\Gamma=\partial D^{+}$, which satisfies the condition (iii). Denote by $E_{p}\left(D^{+}\right), 1<p<\infty$, a Smirnov Banach space of analytic functions in $D^{+}$with the norm

$$
\|f\|_{E_{p}\left(D^{+}\right)}=:\left\|f^{+}\right\|_{L_{p}(\Gamma)}, \quad f \in E_{p}\left(D^{+}\right),
$$


where $f^{+}=f / \Gamma$ are non-tangential boundary values of the function $f$ on $\Gamma$.

Based on the norm (2), we define the weighted Smirnov class. Let $\rho \in$ $L_{1}(\Gamma)$ be some weight function. Define weighted Smirnov class $E_{p, \rho}\left(D^{+}\right)$ by

Let

$$
E_{p, \rho}\left(D^{+}\right) \equiv\left\{f \in E_{1}\left(D^{+}\right):\left\|f^{+}\right\|_{L_{p, \rho}(\Gamma)}<+\infty\right\} .
$$

$$
\|f\|_{E_{p, \rho}\left(D^{+}\right)}=\left\|f^{+}\right\|_{L_{p, \rho}(\Gamma)} .
$$

Similarly we define the Smirnov classes in an unbounded domain. Let $D^{-} \subset \mathbb{C}$ be an unbounded domain containing infinitely remote point $(\infty)$. Denote by ${ }_{m} E_{1}\left(D^{-}\right)$a class of functions from $E_{1}\left(D^{-}\right)$which are analytic in $D^{-}$and have an order $k \leq m$ at infinity, i.e., the function $f \in E_{1}\left(D^{-}\right)$has a Laurent decomposition $f(z)=\sum_{k=-\infty}^{m} a_{k} z^{k}$ in the vicinity of the infinitely remote point $z=\infty$, where $m$ is some integer.

For a given weight function $\rho \in L_{1}(\Gamma)$, the weighted class ${ }_{m} E_{p, \rho}\left(D^{-}\right)$is defined as

with

$$
{ }_{m} E_{p, \rho}\left(D^{-}\right) \equiv\left\{f \in \in_{m} E_{1}\left(D^{-}\right):\left\|f^{-}\right\|_{L_{p, \rho}(\Gamma)}\right\}
$$

$$
\|f\|_{m E_{p, \rho}\left(D^{-}\right)}=\left\|f^{-}\right\|_{L_{p, \rho}(\Gamma)},
$$

where $f^{-}$are non-tangential boundary values of the function $f$ on $\Gamma$.

We will also use some results of $[23,24,25,27]$ related to the solvability of homogeneous and nonhomogeneous Riemann problem in weighted Smirnov classes. Consider the following homogeneous Riemann problem in weighted Smirnov classes:

$$
F^{+}(\xi)-G(\xi) F^{-}(\xi)=0, \text { a.e. } \xi \in \Gamma .
$$

By the solution of the problem (3) we mean a pair of analytic functions

$$
\left(F^{+} ; F^{-}\right) \in E_{p, \rho}\left(D^{+}\right) \times_{m} E_{p, \rho}\left(D^{-}\right),
$$

whose non-tangential boundary values $F^{ \pm}(\xi)$ satisfy the equality (3) a.e. on $\Gamma$. In weightless case, this problem has been well enough studied in the monograph by Danilyuk [10].

In construction of a general solution of homogeneous Riemann problem, an important role is played by the following lemma of uniqueness of the solution for a simplest homogeneous problem.

Lemma C (see [10]). Assume that $D^{+}$is an arbitrary domain bounded by the rectifiable curve $\Gamma$. A homogeneous problem

$$
\Phi^{+}(\xi)-\Phi^{-}(\xi)=0, \quad \xi \in \Gamma,
$$

in a class of functions $\Phi(\cdot)$, belonging to Smirnov classes $E_{1}\left(D^{ \pm}\right) \quad\left(D^{-}=\right.$ $\left.\mathbb{C} \backslash \bar{D}^{+}\right)$and having a finite order $k$ at infinity, admits only trivial solutions in the form of polynomials, whose degree does not exceed $k$. 
Let $S$ be a length of the curve $\Gamma$ and let $z=z(s), 0 \leq s \leq S$, be a parametric representation of $\Gamma$ with respect to the length of the arc $s$. Rewrite the problem (3) as follows:

$$
F^{+}[z(s)]-G(z(s)) F^{-}[z(s)]=0 \text {, a.e. } s \in[0, S] .
$$

Let $\Omega(s) \equiv \theta(z(s)), 0 \leq s \leq S$, and suppose

$$
h_{k}=\Omega\left(s_{k}+0\right)-\Omega\left(s_{k}-0\right), k \in \overline{1, r} ; h_{0}=\Omega(+0)-\Omega(S-0),
$$

where $\xi_{k}=z\left(s_{k}\right), 0<s_{k}<S$, and $a=z(0)=z(S)$ are discontinuity points of the argument $\Omega(\cdot)$. Consider the piecewise holomorphic functions

$$
\begin{gathered}
Z_{(1)}(z)=\exp \left\{\frac{1}{2 \pi i} \int_{\Gamma} \ln |G(z(s))| \frac{d z(s)}{z(s)-z}\right\}, \\
\tilde{Z}_{\theta}(z)=\exp \left\{\frac{1}{2 \pi} \int_{\Gamma} \Omega(s) \frac{d z(s)}{z(s)-z}\right\}=\exp \left\{\frac{1}{2 \pi} \int_{\Gamma} \theta(z(s)) \frac{d z(s)}{z(s)-z}\right\}, z \notin \Gamma .
\end{gathered}
$$

As the argument $\theta(\cdot)$ is defined ambiguously, it is clear that the value of the function $\tilde{Z}_{\theta}(\cdot)$ depends on the chosen argument. Denote the product of these functions by

$$
Z_{\theta}: Z_{\theta}(z) \equiv Z_{(1)}(z) \tilde{Z}_{\theta}(z), z \in \mathbb{C} \backslash \Gamma \text {. }
$$

Hereinafter, the function $Z_{\theta}(\cdot)$ will be called a canonical solution of the problem (3) corresponding to the argument $\theta(\cdot)$.

The following lemma is true for the first multiplier $Z_{(1)}(z)$.

Lemma 1 (see [10]). Let the conditions (i)-(iii) be satisfied for the coefficient $G(\cdot)$ and the curve $\Gamma$. Then the functions $Z_{(1)}(z)$ and $Z_{(1)}^{-1}(z)$ are bounded in each of the domains $D^{ \pm}$.

Represent the function $\Omega(s)$ in the form

$$
\Omega(s)=\Omega_{0}(s)+\Omega_{1}(s), \quad 0 \leq s \leq S,
$$

where $\Omega_{0}(s)$ is a continuous part, and $\Omega_{1}(s)$ is a jump function defined by

$$
\begin{gathered}
\Omega_{1}(0)=0, \\
\Omega_{1}(s)=[\Omega(+0)-\Omega(0)]+\sum_{0<s_{k}<s} h_{k}+[\Omega(s)-\Omega(s-0)], \quad 0<s<S .
\end{gathered}
$$

Denote

$$
h_{0}^{(0)}=\Omega_{0}(S)-\Omega_{0}(0), \quad h_{0}^{(1)}=\Omega_{1}(+0)-\Omega_{1}(S-0) .
$$

Let

and

$$
Z_{(2)}(z)=\exp \left\{\frac{1}{2 \pi} \int_{\Gamma} \Omega_{0}(s) \frac{d z(s)}{z(s)-z}\right\}
$$

$$
Z_{(3)}(z)=\exp \left\{\frac{1}{2 \pi} \int_{\Gamma} \Omega_{1}(s) \frac{d z(s)}{z(s)-z}\right\} \text {. }
$$


It was proved in [10] that the following inclusion is true:

$$
\tilde{Z}_{(2)}^{ \pm 1}(s)=|z(s)-z(0)|^{ \pm \frac{h_{0}^{(0)}}{2 \pi}}\left|Z_{(2)}^{ \pm}[z(s)]\right|^{ \pm 1} \in L_{q}(\Gamma), \quad q \in(0,+\infty) .
$$

The modulus of boundary values of the function $Z_{(3)}(\cdot)$ can be represented by

$$
\left|Z_{(3)}^{+}[z(\sigma)]\right| \equiv|z(0)-z(\sigma)|^{-\frac{h_{0}^{(1)}}{2 \pi}} \prod_{0<s_{k}<S}\left|z\left(s_{k}\right)-z(\sigma)\right|^{-\frac{h_{k}}{2 \pi}},
$$

which follows directly from the lemma below.

Lemma 2 (see [10]). Let the curve $\Gamma$ satisfy the condition (iii) and let $\Omega_{1}(s)$ be an arbitrary jump function with the jumps $h_{0}^{(1)}=\Omega_{1}(+0)-\Omega_{1}(S-0)$ at the point $z(0)$. Then the modulus of boundary values of the function $Z_{(3)}(\cdot)$ can be represented by the formula (4) for a.e. $\sigma \in[0, S]$.

Let us introduce the weight function

$$
\sigma(s) \equiv|z(0)-z(s)|^{-\frac{h_{0}}{2 \pi}} \prod_{0<s_{k}<S}\left|z\left(s_{k}\right)-z(s)\right|^{-\frac{h_{k}}{2 \pi}} .
$$

Let $\rho: \Gamma \rightarrow(0,+\infty)$ be some weight function. Assume that there exist $p_{1}, p_{2} \in(1,+\infty)$ such that

$$
\begin{gathered}
\int_{0}^{S} \sigma^{p p_{1}}(s) \rho^{p_{1}}(z(s)) d s<+\infty . \\
\int_{0}^{S} \sigma^{-q p_{2}}(s) \rho^{-\frac{q}{p} p_{2}}(z(s)) d s<+\infty
\end{gathered}
$$

The following theorem is true.

Theorem 1 (see $[23,27]$ ). Let the conditions (i)-(iii) hold for complexvalued functions $G(\cdot)$ and the curve $\Gamma$. Assume that the conditions (5) and (6) are satisfied for the weight function $\rho(\cdot)$. Then the general solution of the homogeneous problem (3) in the classes $E_{p, \rho}\left(D^{+}\right) \times_{m} E_{p, \rho}\left(D^{-}\right)$has a representation

$$
F(z)=Z_{\theta}(z) P_{m}(z),
$$

where $Z_{\theta}(\cdot)$ is a canonical solution corresponding to the argument $\theta(\cdot)$, and $P_{m}(\cdot)$ is an arbitrary polynomial of degree $k \leq m$ (for $m \leq-1$ we assume $\left.P_{m}(z) \equiv 0\right)$.

This theorem has the following direct corollary.

Corollary 1. Let all the conditions of Theorem 1 be satisfied. Then, if $F(\infty)=0$, the problem (3) has only a trivial solution in the classes $E_{p, \rho}\left(D^{+}\right) \times_{m} E_{p, \rho}\left(D^{-}\right)$, i.e., when $m \leq-1$, the problem (3) has only a trivial solution in the classes $E_{p, \rho}\left(D^{+}\right) \times_{m} E_{p, \rho}\left(D^{-}\right)$. 
Consider the following nonhomogeneous Riemann problem:

$$
F^{+}(z(s))-G(z(s)) F^{-}(z(s))=g(z(s)), s \in(0, S),
$$

where $g \in L_{p, \rho}(\Gamma)$ is a given function. By the solution of the problem (7) we mean a pair of functions

$$
\left(F^{+}(z) ; F^{-}(z)\right) \in E_{p, \rho}\left(D^{+}\right) \times_{m} E_{p, \rho}\left(D^{-}\right),
$$

whose boundary values $F^{ \pm}$on $\Gamma$ a.e. satisfy (7). Introduce the weight function

$$
\nu(s)=: \sigma^{p}(s) \rho(z(s)), \quad s \in(0, S) .
$$

We will assume that the weight function $\rho(\cdot)$ satisfies the condition

$$
\int_{\Gamma} \rho^{-\frac{q}{p}}(z(s))|d z(s)|<+\infty .
$$

The following theorem is proved in [25].

Theorem 2. Let the coefficients $G(z(s)), 0 \leq s \leq S$, of the problem (7) satisfy the conditions (i) and (ii). Assume that their arguments are piecewise Hölder functions and the curve $\Gamma=z([0, S])$ satisfies the condition (iii). If the weight function $\nu(\cdot)$ belongs to the Muckenhoupt class $A_{p}(\Gamma), 1<$ $p<+\infty$, and the weight function $\rho(\cdot)$ satisfies the condition (9), then the analytic function

$$
F_{1}(z) \equiv \frac{Z_{\theta}(z)}{2 \pi i} \int_{\Gamma} \frac{g(\xi) d \xi}{Z_{\theta}^{+}(\xi)(\xi-z)}
$$

is a particular solution of the nonhomogeneous Riemann problem (7) in weighted Smirnov classes $E_{p, \rho}\left(D^{+}\right) \times_{-1} E_{p, \rho}\left(D^{-}\right)$.

Using this theorem, the following theorem is established in [26].

Theorem 3. Let the coefficients $G(z(s)), 0 \leq s \leq S$, of the nonhomogeneous problem (7) satisfy the conditions (i) and (ii). Let $\arg G(\cdot)$ be a piecewise Hölder function on $\Gamma$, where the curve $\Gamma=z([0, S])$ satisfies the condition (iii). Assume that the weight function $\nu(\cdot)$ defined by (8) belongs to the Muckenhoupt class $A_{p}(\Gamma), 1<p<+\infty$, and the condition (9) holds, i.e., $\rho^{-\frac{q}{p}} \in L_{1}(\Gamma)$. Then the following assertions are true with regard to the solvability of the problem (7) in the classes $E_{p, \rho}\left(D^{+}\right) \times_{m} E_{p, \rho}\left(D^{-}\right)$:

1) for $m \geq-1$, the problem (7) has a general solution of the form

$$
F(z)=Z_{\theta}(z) P_{m}(z)+F_{1}(z),
$$

where $Z_{\theta}(\cdot)$ is a canonical solution of the corresponding homogeneous problem (3), $P_{m}(z)$ is an arbitrary polynomial of degree $k \leq m$ (for $m=-1$ we assume $\left.P_{m}(z) \equiv 0\right)$, and $F_{1}(\cdot)$ is a particular solution of the nonhomogeneous problem (7) of the form (10); 
2) for $m<-1$, the nonhomogeneous problem (7) is solvable only when the right-hand side $g(\cdot)$ satisfies the orthogonality conditions

$$
\int_{\Gamma} \frac{g(\xi)}{Z_{\theta}^{+}(\xi)} \xi^{k-1} d \xi=0, \quad k \in \overline{1,-m-1},
$$

and the unique solution $F(z)=F_{1}(z)$ is defined by (10).

\section{Main results}

3.1. Power weight. In Theorems 1 and 3, strong restrictions are imposed on the coefficient $G(\cdot)$ and on the weight function. In this section we consider a more general case. First, we consider the case when the weight has a power form. The index of the problem is found. Then a certain class of weights is introduced, depending on the coefficient. The index of the problem is calculated also in the case when the weight has a general form.

Let us first consider the case where the weight $\rho(\cdot)$ has a power form

$$
\rho(s)=\prod_{k=0}^{m_{0}}\left|z(s)-z\left(t_{k}\right)\right|^{\alpha_{k}}, \quad s \in[0, S], t_{0}=0 .
$$

Let the following relation hold:

$$
\frac{\beta_{i}}{p} \notin \mathbb{Z}_{p} \equiv\left\{k-\frac{1}{p}: k \in \mathbb{Z}\right\}, \quad i \in \overline{0, l},
$$

where the numbers $\beta_{k}$ are defined by

$$
\beta_{k}=-\frac{p}{2 \pi} \sum_{i=0}^{r} h_{i} \chi_{T_{k}}\left(s_{i}\right)+\sum_{i=0}^{m_{0}} \alpha_{i} \chi_{T_{k}}\left(t_{i}\right), \quad k \in \overline{0, l} .
$$

Without loss of generality, we assume $0=\sigma_{0}<\sigma_{1}<\ldots<\sigma_{l}<S$, where

$$
\begin{gathered}
\left\{\sigma_{k}\right\}_{0}^{l} \equiv\left\{s_{k}\right\}_{0}^{r} \cup\left\{t_{k}\right\}_{0}^{m_{0}} ; h_{k}=h\left(\sigma_{k}\right)=\theta\left(z\left(\sigma_{k}+0\right)\right)-\theta\left(z\left(\sigma_{k}-0\right)\right), k \in \overline{1, l} ; \\
h_{0}=h\left(\sigma_{0}\right)=\theta(z(S-0))-\theta(z(+0)),
\end{gathered}
$$

are the jumps corresponding to the argument $\theta(\cdot)$ at the points $\sigma_{k}, k \in \overline{0, l}$. Introduce the function

$$
\tilde{\theta}(z(s))= \begin{cases}\theta(z(s)), & 0<s<\sigma_{1}, \\ \theta(z(s))+2 \pi n_{1}, & \sigma_{1}<s<\sigma_{2}, \\ \vdots & \\ \theta(z(s))+2 \pi n_{l}, & \sigma_{l}<s<S,\end{cases}
$$

where $\left\{n_{k}\right\}_{1}^{l} \subset \mathbb{Z}$. It is not difficult to see that

$$
e^{i \tilde{\theta}(z(s))} \equiv e^{i \theta(z(s))}, \quad s \notin\left\{\sigma_{k}: k \in \overline{1, l}\right\} .
$$


We have

Let

$$
h\left(\sigma_{k}\right)=\left\{\begin{array}{ll}
h_{i}, & \sigma_{k}=s_{i}, \\
0, & \sigma_{k} \notin\left\{s_{i}\right\}_{1}^{r},
\end{array} \quad h\left(\sigma_{0}\right)=h_{0} .\right.
$$

$$
\tilde{G}(z(s))=|G(z(s))| e^{i \tilde{\theta}(z(s))}, \quad 0<s<S,
$$

and consider the Riemann boundary value problem

$$
F^{+}(z(s))-\tilde{G}(z(s)) F^{-}(z(s))=g(z(s)), \quad s \in(0, S),
$$

with the coefficient $\tilde{G}(\cdot)$. As $G(z(s)) \equiv \tilde{G}(z(s)), s \in(0, S)$, it is clear that the issues related to the solvability of the problems (7) and (14) in the classes $E_{p, \rho}\left(D^{+}\right) \times{ }_{m} E_{p, \rho}\left(D^{-}\right)$are equivalent to each other. It is not difficult to see that for the problem (14) the relation (13) becomes

$$
\tilde{\beta}_{k}=-\frac{p}{2 \pi} \tilde{h}_{k}+\sum_{i=0}^{m_{0}} \alpha_{i} \chi_{T_{k}}\left(t_{i}\right), \quad k \in \overline{0, l},
$$

where $\tilde{h}_{k}$ is a jump of the function $\tilde{\theta}(z(s))$ at discontinuity point $s=\sigma_{k}$, i.e.,

$$
\tilde{h}_{k}=\tilde{\theta}\left(z\left(\sigma_{k}+0\right)\right)-\tilde{\theta}\left(z\left(\sigma_{k}-0\right)\right), \quad k \in \overline{1, l} .
$$

We have

$$
\begin{gathered}
\tilde{h}_{1}=h\left(\sigma_{1}\right)+2 \pi n_{1} ; \quad \tilde{h}_{k}=h\left(\sigma_{k}\right)+2 \pi\left(n_{k}-n_{k-1}\right), \quad k \in \overline{2, l} ; \\
\tilde{h}_{0}=h\left(\sigma_{0}\right)-2 \pi n_{l} .
\end{gathered}
$$

Considering these relations for $\tilde{\beta}_{k}$, we obtain

$$
\tilde{\beta}_{1}=\beta_{1}-p n_{l} ; \quad \tilde{\beta}_{k}=\beta_{k}-p\left(n_{k}-n_{k-1}\right), \quad k \in \overline{2, l} ; \quad \tilde{\beta}_{0}=\beta_{0}+p n_{l} .
$$

Consequently,

$$
\begin{gathered}
-\frac{\tilde{\beta}_{1}}{p}=-\frac{\beta_{1}}{p}+n_{1} ; \quad-\frac{\tilde{\beta}_{k}}{p}=-\frac{\beta_{k}}{p}+n_{k}-n_{k-1}, \quad k \in \overline{2, l} ; \\
-\frac{\tilde{\beta}_{0}}{p}=-\frac{\beta_{0}}{p}-n_{l} .
\end{gathered}
$$

Now let us define the integers $n_{k}, k \in \overline{1, l}$, from the inequalities

$$
\left.\begin{array}{l}
-\frac{1}{q}<-\frac{\beta_{1}}{q}+n_{1}<\frac{1}{p}, \\
-\frac{1}{q}<-\frac{\beta_{k}}{p}+n_{k}-n_{k-1}<\frac{1}{p}, \quad k \in \overline{2, l .}
\end{array}\right\}
$$

From the conditions $\left\{\frac{\beta_{k}}{p}\right\}_{1}^{l} \cap \mathbb{Z}_{p}=\emptyset$ it follows that the integers $n_{k}, k \in \overline{1, l}$, are defined uniquely by (15). Applying Theorem 3 to the problem (14), we arrive at the following statement. 
Statement 3. Let the coefficient $G(\cdot)$ and the curve $\Gamma$ satisfy the conditions (i)-(iii), the weight function $\rho(\cdot)$ is defined by (11), and $\left\{\beta_{k}\right\}_{0}^{l}$ is defined by (13). Assume that $\left\{\frac{\beta_{k}}{p}\right\}_{0}^{l} \cap \mathbb{Z}_{p}=\emptyset$ and the integers $n_{k}, k \in \overline{1, l}$, are defined by (15). If the conditions

$$
-\frac{1}{q}<-\frac{\beta_{0}}{p}-n_{l}<\frac{1}{p} ; \quad \alpha_{k}<\frac{p}{q}, \quad k \in \overline{0, m_{0}},
$$

hold, then the assertions 1) and 2) of Theorem 3 are true with regard to the solvability of the problem (7) in the classes $\mathrm{E}_{p, \rho}\left(D^{+}\right) \times{ }_{m} \mathrm{E}_{p, \rho}\left(D^{-}\right)$.

Now we consider the case, where $\beta_{0}$ may not satisfy (16). So let us assume that the coefficient $G(\cdot)$ and the curve $\Gamma$ satisfy all the conditions of Statement 3 except (16). The number $\beta_{0}$, corresponding to the function $\theta(\cdot)$, has the form

$$
\begin{aligned}
\beta_{0}=- & \frac{p}{2 \pi} h_{0}+\alpha_{0}=-\frac{p}{2 \pi}(\theta(a+0)-\theta(a-0))+\alpha_{0} \\
= & -\frac{p}{2 \pi}(\theta(z(+0))-\theta(z(S-0)))+\alpha_{0} .
\end{aligned}
$$

Let $z_{0} \in D^{+}$be an arbitrary fixed point. Consider the following analytic function in $D^{-}$:

$$
\omega(z)=\left(z-z_{0}\right)^{\phi}, \quad z \in D^{-},
$$

where $\phi \in \mathbb{Z}$ is some integer. We have $\omega^{-}(\xi)=\left(\xi-z_{0}\right)^{\phi}, \xi \in \Gamma$. Let $\gamma(\xi)=\arg \omega^{-}(\xi), \xi \in \Gamma$, and

$$
G_{1}(\xi)=\tilde{G}(\xi) \omega^{-}(\xi), \quad \xi \in \Gamma .
$$

It is not difficult to see that the function $G_{1}(\cdot)$ satisfies the conditions (i) and (ii). We have

$$
\theta_{1}(\xi)=\arg G_{1}(\xi)=\tilde{\theta}(\xi)+\gamma(\xi), \quad \xi \in \Gamma .
$$

It is absolutely clear that the discontinuity points and the jumps of the function $\theta_{1}(z(\cdot))$ coincide with the discontinuity points and the jumps of the function $\tilde{\theta}(z(\cdot))$ on $(0, S)$. The jump $h_{0}\left(\theta_{1}\right)$ of the function $\theta_{1}(\cdot)$ at the point $\xi=a=z(0)=z(S)$ is equal to

$$
\begin{gathered}
h_{0}\left(\theta_{1}\right)=\theta_{1}(z(+0))-\theta_{1}(z(S-0)) \\
=\tilde{\theta}(z(+0))-\tilde{\theta}(z(S-0))+\gamma(z(+0))-\gamma(z(S-0))=\tilde{h}_{0}-2 \pi \phi .
\end{gathered}
$$

It is obvious that the quantity $h_{0}\left(\theta_{1}\right)$ does not depend on the choice of the point $z_{0} \in D^{+}$. The quantities $\beta_{k}{ }^{(1)}$, defined by (13) with regard to the coefficient $G_{1}(\cdot)$, are equal to

$$
\beta_{k}^{(1)}=\tilde{\beta}_{k}, \quad k=\overline{1, l ;} \quad \beta_{0}^{(1)}=-\frac{p}{2 \pi} h_{0}\left(\theta_{1}\right)=-\frac{p}{2 \pi} \tilde{h}_{0}+p \phi=\tilde{\beta}_{0}+p \phi .
$$


Consequently

$$
-\frac{\beta_{0}{ }^{(1)}}{p}=-\frac{\tilde{\beta}_{0}}{p}-\phi=-\frac{\beta_{0}}{p}-n_{l}-\phi .
$$

Now define the number $\phi \in \mathbb{Z}$ from the condition $-\frac{1}{q}<-\frac{\beta_{0}{ }^{(1)}}{p}<\frac{1}{p}$, i.e., set

$$
\phi=\left[-\frac{\beta_{0}}{p}+\frac{1}{q}-n_{l}\right] .
$$

Then we obtain that the coefficient $G_{1}(\cdot)$ satisfies all the conditions of Statement 3 , and therefore all the conditions of Theorem 3. Modify the problem (7) in the following way:

$$
F^{+}(\xi)-G(\xi) \omega^{-}(\xi) \frac{F^{-}(\xi)}{\omega^{-}(\xi)}=g(\xi), \quad \xi \in \Gamma,
$$

or

$$
\Phi^{+}(\xi)-G_{1}(\xi) \Phi^{-}(\xi)=g(\xi), \xi \in \Gamma
$$

where

$$
\Phi(z)= \begin{cases}F(z), & z \in D^{+} \\ \frac{F(z)}{\omega(z)}, & z \in D^{-}\end{cases}
$$

It is absolutely clear that

$$
\Phi^{-}(\xi) \sim F^{-}(\xi) \omega^{-1}(\xi), \xi \in \Gamma .
$$

Moreover, if $F(z)$ has an expansion in a neighborhood of $z=\infty$ of the form

$$
F(z)=\sum_{k=-\infty}^{m} a_{k} z^{k}, a_{m} \neq 0, z \rightarrow \infty
$$

then $\Phi(z)$ has an expansion of the form

$$
\Phi(z)=\sum_{k=-\infty}^{m-\phi} b_{k} z^{k}, b_{m-\phi} \neq 0, \quad z \rightarrow \infty .
$$

It follows directly from these relations that the function $F(\cdot)$ belongs to the class ${ }_{m} E_{p, \rho}\left(D^{-}\right)$if and only if the function $\Phi(\cdot)$ belongs to the class ${ }_{m-\phi} E_{p, \rho}\left(D^{-}\right)$, i.e., if the relation

$$
F(\cdot) \in_{m} E_{p, q}\left(D^{-}\right) \Leftrightarrow \Phi(\cdot) \in_{m-\phi} E_{p, q}\left(D^{-}\right),
$$

holds.

Thus, the solvability of the problem (7) in the classes $E_{p, \rho}\left(D^{+}\right) \times$ ${ }_{m} E_{p, \rho}\left(D^{-}\right)$is equivalent to the solvability of the problem (17) in the classes $E_{p, \rho}\left(D^{+}\right) \times{ }_{m-\phi} E_{p, \rho}\left(D^{-}\right)$. As all the conditions of Theorem 3 are satisfied with regard to the problem (17), applying this theorem we obtain the following result. 
Statement 4. Let the coefficient $G(\cdot)$ and the curve $\Gamma$ satisfy the conditions (i)-(iii), and let the weight function $\rho(\cdot)$ be defined by (11). Assume that $\beta_{k}, k \in \overline{0, l}$, defined by (13) satisfy the condition $\left\{\beta_{k}\right\}_{0}^{l} \cap \mathbb{Z}_{p}=\emptyset$, and $\alpha_{k}<\frac{p}{q}, k \in \overline{1, m_{0}}$. Let $\phi=\left[-\frac{\beta_{0}}{p}+\frac{1}{q}-n_{l}\right]$, where the integer $n_{l}$ is defined from the inequalities (15). Then the following assertions are true with regard to the solvability of the problem (17) in the classes $E_{p, \rho}\left(D^{+}\right) \times_{m-\phi} E_{p, \rho}\left(D^{-}\right)$:

(1) for $m-\phi \geq-1$, the problem (17) has a general solution of the form

$$
\Phi(z)=Z_{\theta_{1}}(z) P_{m-\phi}(z)+\Phi_{1}(z),
$$

where $Z_{\theta_{1}}(\cdot)$ is a canonical solution of the homogeneous problem with the coefficient $G_{1}(\cdot), P_{m-\phi}(\cdot)$ is an arbitrary polynomial of degree $k \leq m-\phi$ (for $m-\phi=-1$ we assume $P_{m-\phi}(z) \equiv 0$ ), and $\Phi_{1}(\cdot)$ is a particular solution of the nonhomogeneous problem (17) of the form

$$
\Phi_{1}(z)=\frac{Z_{\theta_{1}}(z)}{2 \pi i} \int_{\Gamma} \frac{g(\xi)}{Z_{\theta_{1}}^{+}(\xi)} \frac{d \xi}{\xi-z}, \quad z \notin \Gamma
$$

(2) for $m-\phi<-1$, the nonhomogeneous problem (17) is solvable only when the right-hand side $g(\cdot)$ satisfies the orthogonality conditions

$$
\int_{\Gamma} \frac{g(\xi)}{Z_{\theta_{1}}^{+}(\xi)} \xi^{k-1} d \xi=0, \quad k \in \overline{1, \phi-m-1},
$$

and the unique solution $\Phi(z)=\Phi_{1}(z)$ is defined by (18).

We call the integer $\phi(G)=m-\phi+1=m-\sum_{k=0}^{l}\left[-\frac{\beta_{k}}{p}+\frac{1}{p}\right]+1$ an index of the problem (7) in the classes $E_{p, \rho}\left(D^{+}\right) \times{ }_{m} E_{p, \rho}\left(D^{-}\right)$.

Thus, for $\phi(G) \geq 0$ the problem (7) is always solvable, while for $\phi(G)<0$ it is only solvable when the orthogonality conditions hold $(-\phi(G))$.

Let us simplify the formulation of Statement 3. From (15) we have

$$
\begin{gathered}
0<\frac{\beta_{1}}{p}+\frac{1}{p}-n_{1}<1 \Rightarrow n_{1}=\left[\frac{\beta_{1}}{p}+\frac{1}{p}\right] \\
0<\frac{\beta_{k}}{p}+\frac{1}{p}-\left(n_{k}-n_{k-1}\right)<1 \Rightarrow n_{k}-n_{k-1}=\left[\frac{\beta_{k}}{p}+\frac{1}{p}\right] \\
\Rightarrow n_{2}=\left[\frac{\beta_{2}}{p}+\frac{1}{p}\right]+n_{1}=\left[\frac{\beta_{1}}{p}+\frac{1}{p}\right]+\left[\frac{\beta_{2}}{p}+\frac{1}{p}\right], \ldots, \\
n_{l}=\sum_{k=1}^{l}\left[\frac{\beta_{k}}{p}+\frac{1}{p}\right] .
\end{gathered}
$$

Then from (16) it follows that

$$
n_{l}=-\left[\frac{\beta_{0}}{p}+\frac{1}{p}\right] \Rightarrow \sum_{k=0}^{l}\left[\frac{\beta_{k}}{p}+\frac{1}{p}\right]=0 .
$$


Therefore, Statement 3 can be restated as follows.

Statement 5. Let the coefficient $G(\cdot)$ and the curve $\Gamma$ satisfy the conditions (i)-(iii), let the weight $\rho(\cdot)$ be defined by (11), and let $\left\{\beta_{k}\right\}_{0}^{l}$ be defined by (13). Let also the conditions

$$
\left\{\frac{\beta_{k}}{p}\right\}_{0}^{l} \cap \mathbb{Z}_{p}=\emptyset ; \quad \alpha_{k}<\frac{p}{q}, \quad k \in \overline{1, \phi}
$$

hold. If $\sum_{k=0}^{l}\left[\frac{\beta_{k}}{p}+\frac{1}{p}\right]=0$, then the assertions 1) and 2) of Statement 3 are true with regard to the solvability of the problem (7) in the classes $E_{p, \rho}\left(D^{+}\right) \times{ }_{m} E_{p, \rho}\left(D^{-}\right)$.

Now let us consider the more general case. By Statement 4 we have

$$
\begin{gathered}
-\frac{1}{p}<\frac{\beta_{0}(1)}{p}<\frac{1}{q} \Rightarrow 0<\frac{\beta_{0}}{p}+n_{l}+\phi+\frac{1}{p}<1 \\
\Rightarrow n_{l}+\phi=-\left[\frac{\beta_{0}}{p}+\frac{1}{p}\right] \Rightarrow \phi=-\left[\frac{\beta_{0}}{p}+\frac{1}{p}\right]-n_{l}=-\sum_{k=0}^{l}\left[\frac{\beta_{k}}{p}+\frac{1}{p}\right] .
\end{gathered}
$$

The solution of the boundary value problem (7) is expressed in terms of the solutions of the boundary value problem (17) by the formula

$$
F(z)= \begin{cases}\Phi(z), & z \in D^{+} \\ \omega(z) \Phi(z), & z \in D^{-} .\end{cases}
$$

Taking into account these relations, from Statement 4 we obtain the following theorem.

Theorem 4. Let the coefficient $G(\cdot)$ and the curve $\Gamma$ satisfy the conditions (i)-(iii), and let the weight function $\rho(\cdot)$ be defined by (11). Assume that the following conditions hold:

$$
\left\{\beta_{k}\right\}_{0}^{l} \cap \mathbb{Z}_{p}=\emptyset ; \quad \alpha_{k}<\frac{p}{q}, \quad k \in \overline{0, m_{0}},
$$

where $\beta_{k}, k \in \overline{0, l}$, are defined by (13). Let

$$
\phi(G)=m-\phi+1=m-\sum_{k=0}^{l}\left[\frac{\beta_{k}}{p}+\frac{1}{p}\right]+1 .
$$

Then the following assertions are true with regard to the solvability of the problem $(7)$ in the classes $E_{p, \rho}\left(D^{+}\right) \times{ }_{m} E_{p, \rho}\left(D^{-}\right)$:

(1) for $\phi(G)=m-\phi+1 \geq 0$, the problem (7) has a general solution of the form

$$
F(z)=F_{0}(z) P_{\phi(G)-1}(z)+F_{1}(z)
$$




$$
F_{0}(z)= \begin{cases}Z_{\theta_{1}}(z), & z \in D^{+}, \\ \left(z-z_{0}\right)^{\phi} Z_{\theta_{1}}(z), & z \in D^{-},\end{cases}
$$

where $Z_{\theta_{1}}(\cdot)$ is a canonical solution of the homogeneous Riemann problem with the coefficient $G_{1}(\cdot), P_{\phi(G)-1}(\cdot)$ is an arbitrary polynomial of degree $k \leq \phi(G)-1\left(\right.$ for $\phi(G)=0$ we assume $\left.P_{\phi(G)-1}(z) \equiv 0\right)$, and $F_{1}(\cdot)$ is a particular solution of the nonhomogeneous problem (7) of the form

$$
F_{1}(z)= \begin{cases}\Phi_{1}(z), & z \in D^{+}, \\ \left(z-z_{0}\right)^{\phi} \Phi_{1}(z), & z \in D^{-},\end{cases}
$$

where the function $\Phi_{1}(\cdot)$ is defined by the Cauchy type integral (18);

(2) for $\phi(G)<0$, the nonhomogeneous problem (7) is solvable only when the right-hand side $g(\cdot)$ satisfies the orthogonality conditions

$$
\int_{\Gamma} \frac{g(\xi)}{Z_{\theta_{1}}^{+}(\xi)} \xi^{k-1} d \xi=0, \quad k \in \overline{1,-\phi(G)},
$$

and the unique solution $F(z)=F_{1}(z)$ is defined by (19).

Consider the case where the argument $\theta(\cdot)$ of the coefficient $G(\cdot)$ is a Hölder function on $\Gamma$. Then the discontinuity points of the function $\theta(\cdot)$ do not exist and, obviously, the corresponding jumps are equal to zero. Thus, by agreed notations, we have $\left\{\sigma_{k}\right\}_{1}^{l} \equiv\left\{t_{k}\right\}_{1}^{m_{0}}$, and $\left\{\tilde{\beta}_{k}\right\}$ 's, corresponding to the argument $\tilde{\theta}(\cdot)$, are equal to

$$
\tilde{\beta}_{k}=-\frac{p}{2 \pi} \tilde{h}_{k}+\alpha_{k} \Rightarrow-\frac{\tilde{\beta}_{k}}{p}=-\frac{\alpha_{k}}{p}+n_{k}-n_{k-1}, \quad k \in \overline{1, m_{0}}, n_{0}=0 .
$$

From (15) we obtain

$$
-\frac{1}{p}<\frac{\alpha_{k}}{p}+n_{k-1}-n_{k}<\frac{1}{q}, \quad \frac{\alpha_{k}}{p}<\frac{1}{q}, k=\overline{1, m_{0}}, n_{0}=0 .
$$

It follows that if there exists $k_{0} \in \overline{1, m_{0}}$ such that $n_{k_{0}-1}-n_{k_{0}}<0$, i.e., $n_{k_{0}-1}-n_{k_{0}} \leq-1$, then

$$
\frac{\alpha_{k_{0}}}{p} \geq-\frac{1}{p}-\left(n_{k_{0}-1}-n_{k_{0}}\right)-\frac{1}{p}+1=\frac{1}{q},
$$

which contradicts the condition $\frac{\alpha_{k_{0}}}{p}<\frac{1}{q}$. Consequently

$$
n_{k-1}-n_{k} \geq 0, \quad k \in \overline{1, m_{0}} \Rightarrow n_{k} \leq n_{k-1} \Rightarrow n_{k} \leq 0, \quad k \in \overline{1, m_{0}},
$$

because $n_{0}=0$. Then $n_{l} \leq 0$. If $n_{l} \leq-1$, then from (16) we obtain

$$
-\frac{1}{p}<\frac{\beta_{0}}{p}+n_{l}<\frac{1}{q}, \frac{\alpha_{0}}{p}<\frac{1}{q},
$$


where $\beta_{0}=\alpha_{0}$. Again we have $\frac{\alpha_{0}}{p}>-\frac{1}{p}-n_{l} \geq \frac{1}{q}$, and this contradicts the condition $\frac{\alpha_{0}}{p}<\frac{1}{q}$. So we obtain $n_{k}=0$ for all $k \in \overline{1, m_{0}}$, and, consequently

$$
-\frac{1}{p}<\frac{\alpha_{k}}{p}<\frac{1}{q}, \quad k \in \overline{1, m_{0}},
$$

i.e., the conditions (16) become the Muckenhoupt condition with regard to the weight function $\rho(\cdot)$. So the following statement is true.

Statement 6. Let the argument $\theta(\cdot)$ of the coefficient $G(\cdot)$ be a Hölder function on $\Gamma$. Then the conditions (16) of Statement 3 hold if and only if the weight $\rho(\cdot)$ satisfies the Muckenhoupt condition, i.e., $\rho(\cdot) \in A_{p}(\Gamma), 1<$ $p<+\infty$.

3.2. General weight. Before turning to the case of a general weight, let us define the class of weights we need. So, let $\left\{\xi_{k}\right\}_{1}^{n} \subset \Gamma$ be some finite subset of $\Gamma$. Denote by $A_{\rho}\left(\left\{\xi_{k}\right\}_{1}^{n} ; \Gamma\right)$ the set of weights $\rho(\cdot)$ for which there exists a set of integers $\left\{n_{k}\right\}_{1}^{n} \subset \mathbb{Z}$ such that $\tilde{\rho}(\cdot) \in A_{p}(\Gamma)$, where

$$
\tilde{\rho}(\xi)=\prod_{k=1}^{n}\left|\xi-\xi_{k}\right|^{-n_{k} p} \rho(\xi), \quad \xi \in \Gamma .
$$

The following lemma is true.

Lemma 3. The set of integers $\left\{n_{k}\right\}_{1}^{n} \subset \mathbb{Z}$ in the definition of the class $A_{p}\left(\left\{\xi_{k}\right\}_{1}^{n} ; \Gamma\right)$ with $1<p<+\infty$ is defined uniquely.

Proof. First we consider the case where the set $\left\{\xi_{k}\right\}_{1}^{n}$ consists of one point, i.e., $n=1$. Assume the contrary, i.e., there exist $n_{1}, n_{2} \in \mathbb{Z}$ such that

$$
\rho_{k}(\xi)=\left|\xi-\xi_{1}\right|^{-p n_{k}} \rho(\xi) \in A_{p}(\Gamma), \quad k=1,2 .
$$

Without loss of generality, we will assume that $n_{2}=n_{1}+n_{0}$, where $n_{0} \in \mathbb{N}$, i.e., $n_{0} \geq 1$. We have

$$
\rho_{2}(\xi)=\rho_{1}(\xi)\left|\xi-\xi_{1}\right|^{-p n_{0}} \Rightarrow\left|\xi-\xi_{1}\right|^{-n_{0}}=\rho_{2}^{\frac{1}{p}}(\xi) \rho_{1}^{-\frac{1}{p}}(\xi) .
$$

Hence, applying Hölder's inequality, we obtain

$$
\int_{\Gamma}\left|\xi-\xi_{1}\right|^{-n_{0}}|d \xi| \leq\left(\int_{\Gamma} \rho_{2}(\xi)|d \xi|\right)^{\frac{1}{p}}\left(\int_{\Gamma} \rho_{1}^{-\frac{q}{p}}(\xi)|d \xi|\right)^{\frac{1}{q}}, \frac{1}{p}+\frac{1}{q}=1 .
$$

From $\rho_{k}(\cdot) \in A_{p}(\Gamma), 1<p<+\infty, k=1,2$, it follows that $\rho_{2}(\cdot) \in$ $L_{1}(\Gamma), \rho_{1}^{-\frac{q}{p}}(\cdot) \in L_{1}(\Gamma)$. Then from $(20)$ we obtain $\left|\xi-\xi_{1}\right|^{-n_{0}} \in L_{1}(\Gamma)$, which contradicts the condition $n_{0} \geq 1$. The case $n=1$ is proved.

The general case is reduced to this case by using the relation

$$
\prod_{k=1}^{n}\left|\xi-\xi_{1}\right|^{-p n_{k}} \rho(\xi) \in A_{p}(\Gamma) \Rightarrow\left|\xi-\xi_{1}\right|^{-p n_{k}} \rho(\xi) \in A_{p}\left(\Gamma_{k}\right), \quad k \in \overline{1, n},
$$


where $\Gamma_{k} \subset \Gamma, k \in \overline{1, n}$, is a part of the curve $\Gamma$, which contains only $\xi_{k}$ and no other point $\xi_{i}, i \neq k$, and, besides, $\xi_{k}$ is an internal point of $\Gamma_{k}$.

As before, we will assume that $0=\sigma_{0}<\sigma_{1}<\ldots<\sigma_{l}<S$, and let

$$
\tilde{\theta}(z(s))= \begin{cases}\theta(z(s)), & 0<s<\sigma_{1}, \\ \theta(z(s))+2 \pi \tilde{n}_{1}, & \sigma_{1}<s<\sigma_{2}, \\ \vdots & \\ \theta(z(s))+2 \pi \tilde{n}_{l}, & \sigma_{l}<s<S,\end{cases}
$$

where $\left\{\tilde{n}_{k}\right\}_{1}^{l} \subset \mathbb{Z}$ are some integers. Let

$$
\tilde{G}(z(s))=|G(z(s))| e^{i \tilde{\theta}(z(s))}, \quad 0<s<S,
$$

and consider the Riemann boundary value problem

$$
F^{+}(z(s))-\tilde{G}(z(s)) F^{-}(z(s))=g(z(s)), \quad s \in(0, S),
$$

with the coefficient $\tilde{G}(\cdot)$. Let $\xi_{k}=z\left(\sigma_{k}\right), k \in \overline{0, l}$. Assume that $\nu(\cdot) \in$ $A_{p}\left(\left\{\xi_{k}\right\}_{0}^{l} ; \Gamma\right)$. Then it follows from Lemma 3 that there exist $\left\{n_{k}\right\}_{0}^{l} \subset \mathbb{Z}$ such that

$$
\prod_{k=0}^{l}\left|z(\cdot)-\xi_{k}\right|^{-p n_{k}} \nu(\cdot) \in A_{p}(\Gamma) .
$$

Let us find the jumps of the functions $\tilde{\theta}(\cdot)$ at the points $\xi_{k}$. We have

$$
\begin{aligned}
& \tilde{h}_{1}=\tilde{\theta}\left(z\left(\sigma_{1}+0\right)\right)-\tilde{\theta}\left(z\left(\sigma_{1}-0\right)\right)=h\left(\sigma_{1}\right)+2 \pi \tilde{n}_{1} ; \\
& \tilde{h}_{k}=h\left(\sigma_{k}\right)+2 \pi\left(\tilde{n}_{k}-\tilde{n}_{k-1}\right), \quad k \in \overline{2, l} ; \\
& \tilde{h}_{0}=\tilde{\theta}(z(S-0))-\tilde{\theta}(z(+0))=h\left(\sigma_{0}\right)-2 \pi \tilde{n}_{l}=h_{0}-2 \pi \tilde{n}_{l} .
\end{aligned}
$$

Similarly to the previous case, the weight function

$$
\tilde{\nu}(s)=\tilde{\sigma}^{p}(s) \rho(z(s))
$$

corresponds to the problem (22), where

$$
\begin{gathered}
\tilde{\sigma}(s)=|z(0)-z(s)|^{-\frac{\tilde{h}_{0}}{2 \pi}} \prod_{0<s_{k}<S}\left|z\left(s_{k}\right)-z(s)\right|^{-\frac{\tilde{h}_{k}}{2 \pi}} \\
=\left|z(s)-\xi_{0}\right|^{-\frac{h_{0}}{2 \pi}}\left|z(s)-\xi_{0}\right|^{\tilde{n}_{l}} \prod_{k=1}^{l}\left|z(s)-\xi_{k}\right|^{-\frac{h_{k}}{2 \pi}}\left|z(s)-\xi_{k}\right|^{-\tilde{n}_{k}+\tilde{n}_{k-1}} \\
=\sigma(s)\left|z(s)-\xi_{0}\right|^{\tilde{n}_{l}} \prod_{k=1}^{l}\left|z(s)-\xi_{k}\right|^{-\tilde{n}_{k}+\tilde{n}_{k-1}},
\end{gathered}
$$


with $\tilde{n}_{0}=0$. Consequently

$$
\tilde{\nu}(s)=\nu(s)\left|z(s)-\xi_{0}\right|^{p \tilde{n}_{l}} \prod_{k=1}^{l}\left|z(s)-\xi_{k}\right|^{-p\left(\tilde{n}_{k}-\tilde{n}_{k-1}\right)} .
$$

Now let $\tilde{n}_{1}=n_{1}, \tilde{n}_{k}-\tilde{n}_{k-1}=n_{k}, k \in \overline{2, l}$. It is absolutely clear that if $\tilde{n}_{l}=-n_{0}$, then $\tilde{\nu}(\cdot) \in A_{p}(\Gamma)$. Therefore, applying Theorem 3 to the problem (22), we obtain the following corollary.

Corollary 2. Let the coefficient $G(\cdot)$ and the curve $\Gamma$ satisfy the conditions (i)-(iii). Assume that $\arg G(\cdot)$ is a piecewise Hölder function on $\Gamma$, the weight function $\nu(\cdot)$ defined by (8) belongs to the class $A_{p}\left(\left\{\xi_{k}\right\}_{0}^{l} ; \Gamma\right)$, $1<p<+\infty$, and $\left\{n_{k}\right\}_{0}^{l} \subset \mathbb{Z}$ are the corresponding integers. We find the integer $\tilde{n}_{l}$ from the relations

$$
\tilde{n}_{k}=n_{k}+\tilde{n}_{k-1}, \quad \tilde{n}_{0}=0 .
$$

If $\tilde{n}_{l}=-n_{0}$ and $\rho^{-\frac{q}{p}}(\cdot) \in L_{1}(\Gamma)$, then the assertions 1) and 2) of Theorem 3 are true with regard to the solvability of the problem (7) in the classes $E_{p, \rho}\left(D^{+}\right) \times{ }_{m} E_{p, \rho}\left(D^{-}\right)$.

Now we consider the general case, i.e., the case where the condition $\tilde{n}_{l}=$ $-n_{0}$ may in general not hold, with $\tilde{n}_{l}$ defined from $(23)$. Let $z_{0} \in D^{+}$be an arbitrary fixed point. Consider the following analytic function in $D^{-}$:

$$
\omega(z)=\left(z-z_{0}\right)^{\phi}, \quad z \in D^{-},
$$

where $\phi \in \mathbb{Z}$ is some integer. Following the previous case (i.e., the case of power weight), we have

$$
\omega^{-}(\xi)=\left(\xi-z_{0}\right)^{\phi}, \gamma(\xi)=\arg \omega^{-}(\xi), \quad \xi \in \Gamma .
$$

Let

$$
G_{1}(\xi)=\tilde{G}(\xi) \omega^{-}(\xi), \quad \xi \in \Gamma .
$$

Consequently

$$
\theta_{1}(\xi)=\arg G_{1}(\xi)=\tilde{\theta}(\xi)+\gamma(\xi), \quad \xi \in \Gamma .
$$

The discontinuity points and the jumps of the function $\theta_{1}(z(\cdot))$ coincide with the discontinuity points and the jumps of the function $\tilde{\theta}(z(\cdot))$ on $(0, S)$. The jump $h_{0}\left(\theta_{1}\right)$ of the function $\theta_{1}(\cdot)$ at the point $\xi=a$ is equal to

$$
\begin{gathered}
h_{0}\left(\theta_{1}\right)=\theta_{1}(z(+0))-\theta_{1}(z(S-0)) \\
=\tilde{\theta}(z(+0))-\tilde{\theta}(z(S-0))+\gamma(z(+0))-\gamma(z(S-0)) \\
=\tilde{h}_{0}-2 \pi \phi=h\left(\sigma_{0}\right)-2 \pi \tilde{n}_{l}-2 \pi \phi .
\end{gathered}
$$

As before, the weight

$$
\nu_{1}(s)=\sigma_{1}^{p}(s) \rho(z(s))
$$


corresponds to the Riemann problem with the coefficient $G_{1}(\cdot)$, where

$$
\begin{gathered}
\tilde{\sigma}_{1}(s)=|z(0)-z(s)|^{-\frac{h_{0}\left(\theta_{1}\right)}{2 \pi}} \prod_{0<s_{k}<S}\left|z\left(s_{k}\right)-z(s)\right|^{-\frac{\tilde{h}_{k}\left(\theta_{1}\right)}{2 \pi}} \\
=\left|z(s)-\xi_{0}\right|^{-\frac{h_{0}}{2 \pi}}\left|z(s)-\xi_{0}\right|^{\tilde{n}_{l}+\phi} \prod_{k=1}^{l}\left|z(s)-\xi_{k}\right|^{-\frac{h_{k}}{2 \pi}}\left|z(s)-\xi_{k}\right|^{-\tilde{n}_{k}+\tilde{n}_{k-1}} \\
=\sigma(s)\left|z(s)-\xi_{0}\right|^{\tilde{n}_{l}+\phi} \prod_{k=1}^{l}\left|z(s)-\xi_{k}\right|^{-\tilde{n}_{k}+\tilde{n}_{k-1}},
\end{gathered}
$$

with $\tilde{n}_{0}=0$. Then

$$
\nu_{1}(s)=\nu(s)\left|z(s)-\xi_{0}\right|^{p\left(\tilde{n}_{l}+\phi\right)} \prod_{k=1}^{l}\left|z(s)-\xi_{k}\right|^{-p\left(\tilde{n}_{k}-\tilde{n}_{k-1}\right)} .
$$

We find the integer $\tilde{n}_{l}$ from (23) and set

$$
\tilde{n}_{l}+\phi=-n_{0} \Rightarrow \phi=-n_{0}-\tilde{n}_{l} .
$$

Then we obtain that the coefficient $G_{1}(\cdot)$ satisfies all the conditions of Theorem 3. Transform the problem (7) as follows:

$$
\begin{gathered}
F^{+}(\xi)-G(\xi) \omega^{-}(\xi) \frac{F^{-}(\xi)}{\omega^{-}(\xi)}=g(\xi), \quad \xi \in \Gamma, \\
\mathbb{1} \\
\Phi^{+}(\xi)-G_{1}(\xi) \Phi^{-}(\xi)=g(\xi), \quad \xi \in \Gamma,
\end{gathered}
$$

where

$$
\Phi(z)= \begin{cases}F(z), & z \in D^{+} \\ \frac{F(z)}{\omega(z)}, & z \in D^{-}\end{cases}
$$

It is absolutely clear that

$$
\Phi^{-}(\xi)=F^{-}(\xi) \omega^{-1}(\xi), \quad \xi \in \Gamma .
$$

Moreover, if $F(z)$ has an expansion in the neighborhood of $z=\infty$ of the form

$$
F(z)=\sum_{k=-\infty}^{m} a_{k} z^{k}, a_{m} \neq 0, z \rightarrow \infty
$$

then $\Phi(z)$ has an expansion of the form

$$
\Phi(z)=\sum_{k=-\infty}^{m-\phi} b_{k} z^{k}, b_{m-\phi} \neq 0, \quad z \rightarrow \infty,
$$

and vice versa. In view of

$$
\omega(\xi) \sim \text { const }, \quad \xi \in \Gamma,
$$


from these relations we obtain that the function $F(\cdot)$ belongs to the class ${ }_{m} E_{p, \rho}\left(D^{-}\right)$if and only if the function $\Phi(\cdot)$ belongs to the class ${ }_{m-\phi} E_{p, \rho}\left(D^{-}\right)$, i.e., $F(\cdot) \in{ }_{m} E_{p, \rho}\left(D^{-}\right) \Leftrightarrow \Phi(\cdot) \in{ }_{m-\phi} E_{p, \rho}\left(D^{-}\right)$. Thus, the solvability of the problem (7) in the classes $E_{p, \rho}\left(D^{+}\right) \times{ }_{m} E_{p, \rho}\left(D^{-}\right)$is equivalent to the solvability of the problem (24) in the classes $E_{p, \rho}\left(D^{+}\right) \times{ }_{m-\phi} E_{p, \rho}\left(D^{-}\right)$. Applying Theorem 3 to the problem (24), we obtain the following statement.

Statement 7. Let the coefficient $G(\cdot)$ and the curve $\Gamma$ satisfy the conditions (i)-(iii), and let $\arg G(\cdot)$ be a piecewise Hölder function on $\Gamma$. Assume that the weight function $\nu(\cdot)$ defined by (8) belongs to the class $A_{p}\left(\left\{\xi_{k}\right\}_{0}^{l} ; \Gamma\right)$, $\left\{n_{k}\right\}_{0}^{l} \subset \mathbb{Z}$ are the corresponding integers, $\phi=-\sum_{k=0}^{l} n_{k}$, and $\rho^{-\frac{q}{p}}(\cdot) \in$ $L_{1}(\Gamma)$. Then the following assertions are true with regard to the solvability of the problem (24) in the classes $E_{p, \rho}\left(D^{+}\right) \times{ }_{m-\phi} E_{p, \rho}\left(D^{-}\right)$:

(1) for $m-\phi \geq-1$, the problem (24) has a general solution of the form

$$
\Phi(z)=Z_{\theta_{1}}(z) P_{m-\phi}(z)+\Phi_{1}(z),
$$

where $Z_{\theta_{1}}(\cdot)$ is a canonical solution of the homogeneous problem with the coefficient $G_{1}(\cdot), P_{m-\phi}(\cdot)$ is an arbitrary polynomial of degree $k \leq m-\phi$ (for $m-\phi=-1$ we assume $P_{m-\phi}(z) \equiv 0$ ), and $\Phi_{1}(\cdot)$ is a particular solution of the form

$$
\Phi_{1}(z)=\frac{Z_{\theta_{1}}(z)}{2 \pi i} \int_{\Gamma} \frac{g(\xi)}{Z_{\theta_{1}}^{+}(\xi)} \frac{d \xi}{\xi-z}, \quad z \notin \Gamma
$$

(2) for $m-\phi<-1$, the nonhomogeneous problem (24) is solvable only when the right-hand side $g(\cdot)$ satisfies the orthogonality conditions

$$
\int_{\Gamma} \frac{g(\xi)}{Z_{\theta_{1}}^{+}(\xi)} \xi^{k-1} d \xi=0, \quad k \in \overline{1, \phi-m-1},
$$

and the unique solution $\Phi_{1}(\cdot)$ is defined by $(25)$.

Now back to the problem (7). Considering the relationship between the problems (7) and (24), we have

$$
F(z)= \begin{cases}\Phi(z), & z \in D^{+} \\ \omega(z) \Phi(z), & z \in D^{-}\end{cases}
$$

So the following theorem is true.

Theorem 5. Let $G(\cdot), \Gamma$, arg $G(\cdot), \nu(\cdot), \phi$, and $\rho^{-\frac{q}{p}}$ be the same as in Statement 7 . Then the following assertions are true with regard to the solvability of the problem (7) in the classes $E_{p, \rho}\left(D^{+}\right) \times{ }_{m} E_{p, \rho}\left(D^{-}\right)$:

(1) for $m-\phi \geq-1$, the problem (24) has a general solution of the form

$$
F_{0}(z)= \begin{cases}Z_{\theta_{1}}(z) P_{m-\phi}(z)+\Phi_{1}(z), & z \in D^{+}, \\ \left(z-z_{0}\right)^{\phi}\left(Z_{\theta_{1}}(z) P_{m-\phi}(z)+\Phi_{1}(z)\right), & z \in D^{-},\end{cases}
$$


where $Z_{\theta_{1}}(\cdot)$ is a canonical solution of homogeneous problem

$$
F^{+}(\xi)-G_{1}(\xi) F^{-}(\xi)=0, \quad \xi \in \Gamma,
$$

$G_{1}(\cdot)$ is a coefficient, $P_{m-\phi}(\cdot)$ is an arbitrary polynomial of degree $k \leq m-\phi$ (for $m-\phi=-1$ we assume $P_{m-\phi}(z) \equiv 0$ ), and $\Phi_{1}(\cdot)$ is defined by $(25)$;

(2) for $m-\phi<-1$, the nonhomogeneous problem (7) is solvable only when the right-hand side $g(\cdot)$ satisfies the orthogonality conditions (26), and the unique solution $F_{1}(\cdot)$ is defined by

$$
F_{1}(z)= \begin{cases}\Phi_{1}(z), & z \in D^{+} \\ \left(z-z_{0}\right)^{\phi} \Phi_{1}(z), & z \in D^{-}\end{cases}
$$

where $\Phi_{1}(\cdot)$ is defined by $(25)$.

The number $\phi(G)=m-\phi+1=m-\sum_{k=0}^{l} n_{k}+1$, where the integers $\left\{n_{k}\right\}_{0}^{l}$ are defined from the condition $\nu(\cdot) \in A_{p}\left(\left\{\xi_{k}\right\}_{0}^{l} ; \Gamma\right)$, is called an index of the problem (7) in the classes $E_{p, \rho}\left(D^{+}\right) \times{ }_{m} E_{p, \rho}\left(D^{-}\right)$.

Thus, for $\phi(G) \geq 0$ the problem (7) is always solvable, for $\phi(G)=0$ it has a unique solution and it is solvable for all $g(\cdot) \in L_{p, \rho}(\Gamma)$; for $\phi(G)<0$ it is solvable if the orthogonality conditions hold $(-\phi(G))$.

Assume that the argument $\theta(\cdot)$ of the coefficient $G(\cdot)$ is a Hölder function on $\Gamma$. In this case, the discontinuity points and the jumps of the function $\theta(\cdot)$ do not exist and, therefore, $\sigma_{k}=t_{k}, k \in \overline{1, m_{0}}$. Let us consider the weight function

$$
\tilde{\nu}(s)=\tilde{\sigma}^{p}(s) \rho(z(s)),
$$

which corresponds to the function $\tilde{\theta}(\cdot)$ defined by $(21)$, where

$$
\tilde{\sigma}(s)=\left|z(s)-\xi_{0}\right|^{\tilde{n}_{m_{0}}} \prod_{k=1}^{l}\left|z(s)-\xi_{k}\right|^{-\tilde{n}_{k}+\tilde{n}_{k-1}}, \quad \tilde{n}_{0}=0 .
$$

Denote by $\Gamma_{k}, k \in \overline{0, m_{0}}$ the connected part of $\Gamma$, for which $O_{\delta}\left(\xi_{k}\right) \subset$ $\Gamma_{k}, \quad \xi_{i} \notin \bar{\Gamma}_{k}$ for any $i \neq k$, where $O_{\delta}\left(\xi_{k}\right)=\left\{\xi \in \Gamma:\left|\xi-\xi_{k}\right|<\delta\right\}$, and $\bar{\Gamma}_{k}$ is a closure of $\Gamma_{k}$. Consequently

$$
\tilde{\sigma}(s)=\tilde{\nu}^{\frac{1}{p}}(s) \rho^{-\frac{1}{p}}(z(s)), \quad 0<s<S .
$$

Assume that all the conditions of Theorem 5 hold. Then

$$
\tilde{\nu}(\cdot) \in A_{p}(\Gamma) \wedge \rho^{-\frac{q}{p}}(\cdot) \in L_{1}(\Gamma)
$$

and so

$$
\tilde{\nu}(\cdot) \in A_{p}\left(\Gamma_{k}\right), \quad k \in \overline{0, m_{0}}
$$

As

$$
\begin{gathered}
\tilde{\sigma}(\xi) \sim\left|\xi-\xi_{k}\right|^{\tilde{n}_{k-1}-\tilde{n}_{k}}, \tilde{n}_{0}=0, \quad \xi \in \Gamma_{k}, k=\overline{1, m_{0}} \\
\tilde{\sigma}(\xi) \sim\left|\xi-\xi_{0}\right|^{\tilde{n}_{m_{0}}}, \quad \xi \in \Gamma_{0}
\end{gathered}
$$


it is clear that

$$
\begin{gathered}
\left|z(s)-\xi_{k}\right|^{\tilde{n}_{k-1}-\tilde{n}_{k}} \sim \tilde{\nu}^{\frac{1}{p}}(s) \rho^{\frac{-1}{p}}(z(s)), z(s) \in \Gamma_{k}, \tilde{n}_{0}=0, k \in \overline{1, m_{0}} ; \\
\left|z(s)-\xi_{0}\right|^{\tilde{n}_{m_{0}}} \sim \tilde{\nu}^{\frac{1}{p}}(s) \rho^{\frac{-1}{p}}(z(s)), \quad z(s) \in \Gamma_{0} .
\end{gathered}
$$

If, for some $k_{0} \in \overline{0, m_{0}}$,

$$
\tilde{n}_{k_{0}-1}-\tilde{n}_{k_{0}} \leq-1\left(k_{0}=0 \Rightarrow \tilde{n}_{m_{0}} \leq-1\right),
$$

then from the previous relations we obtain

$$
\begin{aligned}
& \int_{\Gamma_{k_{0}}}\left|z(s)-\xi_{k_{0}}\right|^{\gamma_{k_{0}}}|d z(s)| \\
& \leq C\left(\int_{\Gamma_{k_{0}}} \tilde{\nu}(s)|d z(s)|\right)^{\frac{1}{p}}\left(\int_{\Gamma_{k_{0}}} \rho^{-\frac{q}{p}}(z(s))|d z(s)|\right)^{\frac{1}{q}},
\end{aligned}
$$

where

$$
\gamma_{k}= \begin{cases}\tilde{n}_{k-1}-\tilde{n}_{k}, & k \in \overline{1, m_{0}}, \\ n_{m_{0}}, & k=0 .\end{cases}
$$

Hence, in view of $(27)$ and (28), we have $\left|\xi-\xi_{k_{0}}\right|^{\gamma_{k_{0}}} \in L_{1}\left(\Gamma_{k_{0}}\right)$, which contradicts the condition $\gamma_{k_{0}} \leq-1$. Consequently, $\gamma_{k} \geq 0$ for all $k \in \overline{0, m_{0}}$. We have

$$
\tilde{n}_{k-1}-\tilde{n}_{k} \geq 0, \quad k \in \overline{1, m_{0}} \Rightarrow \tilde{n}_{k} \leq \tilde{n}_{k-1}, \tilde{n}_{0}=0, \quad k \in \overline{1, m_{0}} .
$$

It follows directly that if for some $k_{0} \in \overline{1, m_{0}-1}$ the relation $\tilde{n}_{k_{0}} \leq-1$ holds, then $\tilde{n}_{m_{0}} \leq-1$. As a result, from (29) when $k_{0}=m_{0}$ we arrive at a contradiction. It follows that, in the case considered, all conditions of Theorem 5 can be satisfied only when

$$
\tilde{n}_{k}=0, \quad k \in \overline{0, m_{0}} \Rightarrow \tilde{\nu}(s)=\rho(z(s)) \Rightarrow \rho(\cdot) \in A_{p}(\Gamma) .
$$

So, the following statement is valid.

Statement 8. Let the argument $\theta(\cdot)$ of the coefficient $G(\cdot)$ be a Hölder function on $\Gamma$. Then the conditions of Theorem 5 are satisfied, i.e., $\nu(\cdot) \in$ $A_{p}\left(\left\{t_{k}\right\}_{0}^{m_{0}} ; \Gamma\right), 1<p<+\infty$, if and only if $\rho(\cdot) \in A_{p}(\Gamma)$.

Remark 1. It follows from the results of Statements 6 and 7, and the expression $\nu(s)=\sigma^{p}(s) \rho(z(s))$ for the weight function $\nu(\cdot)$ that, when $\arg G(\cdot)$ has discontinuities, then for solvability of corresponding problems, the weight $\rho(\cdot)$ may in general not satisfy the Muckenhoupt condition. 


\section{Acknowledgements}

This work is supported by the Scientific and Technological Research Council of Turkey (TUBITAK) with Azerbaijan National Academy of Sciences (ANAS), Project Number: 19042020 and by the Science Development Foundation under the President of the Republic of Azerbaijan - Grant No. EIFBGM-4-RFTF1/2017- 21/02/1-M-19

The authors thank the anonymous referees for their careful reading the manuscript, for comments and suggestions.

\section{References}

[1] B. T. Bilalov, The basis property of some systems of exponentials of cosines and sines, Differ. Uravn. 26(1) (1990), 10-16. (Russian)

[2] B. T. Bilalov, On the basis property of the system $\left\{e^{i n x} \operatorname{sinn} x\right\}_{1}^{\infty}$ and of a system of exponentials with shift, Dokl. Akad. Nauk, 345(2) (1995), 644-647. (Russian)

[3] B. T. Bilalov, Basis properties of some systems of exponentials, cosines, and sines, Sibirsk. Mat. Zh. 45(2) (2004), 264-273. (Russian)

[4] B. T. Bilalov, Basis properties of power systems in $L_{p}$, Sibirsk. Mat. Zh. 47(1) (2006), 1-12. (Russian)

[5] B. T. Bilalov, A system of exponentials with shift and a problem of A. G. Kostyuchenko, Sibirsk. Mat. Zh. 50(2) (2009), 279-288. (Russian)

[6] B. T. Bilalov, On the solution of a problem of A. G. Kostyuchenko, Sibirsk. Mat. Zh. 53(3) (2012), 509-526. (Russian)

[7] B. T. Bilalov, On the basis property of a perturbed system of exponentials in Morrey type spaces, Sibirsk. Mat. Zh. 60(2) (2019), 323-350. (Russian)

[8] B. T. Bilalov, T. B. Gasymov, and A. A. Guliyeva, On the solvability of the Riemann boundary value problem in Morrey-Hardy classes, Turkish J. Math. 40(50) (2016), $1085-1101$.

[9] R. R. Coifman and C. Fefferman, Weighted norm inequalities for maximal functions and singular integrals, Studia Math. 51(3) (1974), 241-249.

[10] I. I. Danilyuk, Irregular Boundary Value Problems on Half Plane, Nauka, Moscow, 1975. (Russian)

[11] G. David, Operateurs integraux singulars sur certains courbes du plan complexe, Ann. Sci. Ec. Norm. Super. 17 (1984), 157-189.

[12] E. M. Dynkin, Methods of theory of singular integrals, in: Current Problems in Mathematics. Fundamental Directions, Vol. 15, Akad. Nauk SSSR, Vsesoyuz. Inst. Nauchn. i Tekhn. Inform., Moscow, 1987, pp. 197-292. (Russian)

[13] F. D. Gakhov, Boundary Value Problems, Nauka, Moscow, 1977. (Russian)

[14] V. M. Kokilashvili, Boundary value problems of analytic and harmonic functions in a domain with piecewise smooth boundary in the frame of variable exponent Lebesgue spaces, in: Modern Aspects of the Theory of Partial Differential Equations, Oper. Theory Adv. Appl. 216, Birkhäuser/Springer Basel AG, Basel, (2011), pp. 17-39.

[15] G. Litvinchuk, Boundary-Value Problems and Singular Integral Equations with Shift, Nauka, Moscow, 1977. (Russian)

[16] G. Manjavidze and N. Manjavidze, Boundary value problems for analytic and generalized analytic functions, J. Math. Sci. 160 (2009), 745-821.

[17] Z. Meshveliani, The Riemann-Hilbert problem in weighted Smirnov classes of analytic functions, Proc. A. Razmadze Math. Inst. 137 (2005), 65-86. 
[18] E.I. Moiseev, The basis property for systems of sines and cosines, Dokl. Akad. Nauk SSSR 275(4) (1984), 794-798. (Russian)

[19] E.I. Moiseev, On the basis property of a system of sines, Differ. Uravn. 23(1) (1987), 177-179. (Russian)

[20] N. Muskhelishvili, Singular Integral Equations, Noordhoff, Groningen-Leyden, 1953.

[21] T. I. Najafov and N. P. Nasibova, On the Noetherness of the Riemann problem in generalized weighted Hardy classes, Azerb. J. Math. 5(2) (2015), 109-139.

[22] I. Privalov, Boundary Properties of Analytic Functions, Gosudarstv. Izdat. Tehn.Teor. Lit., Moscow - Leningrad, 1950. (Russian)

[23] S. R. Sadigova, The general solution of the homogeneous Riemann problem in the weighted Smirnov classes, Proc. Inst. Math. Mech. Natl. Acad. Sci. Azerb. 40(2) (2014), $115-124$.

[24] S. R. Sadigova, The general solution of the homogeneous Riemann problem in weighted Smirnov classes with general weight, Azerb. J. Math. 9(2) (2019), 134-147

[25] S. R. Sadigova, On particular solution of the nonhomogeneous Riemann problem in the weighted Smirnov classes with the general weight, Proc. Inst. Math. Mech. Natl. Acad. Sci. Azerb. 46(2) (2020), 272-283.

[26] S. R. Sadigova, On Riemann problem in weighted Smirnov classes with power weight, Rocky Mountain J. Math. 2021 (accepted).

[27] S. R. Sadigova and A. E. Guliyeva, On the solvability of Riemann-Hilbert problem in the weighted Smirnov classes, Anal. Math. 44(4) (2018), 587-603.

[28] I. N. Vekua, New Methods of the Solutions of Elliptic Equations, Gostekhizdat, Moscow, 1948. (Russian)

[29] I. N. Vekua, Generalized Analytic Functions, Pergamon Press, Oxford, 1962.

[30] I. N. Vekua, Systems of Singular Integral Equations, Nauka, Moscow, 1970. (Russian)

[31] V. I. Vlasov, Hardy spaces, approximation issues and boundary value problems, Eurasian Math. J. 9(3) ( 2018), 85-94.

[32] V. I. Vlasov and A. V. Rachkov, Weighted Hardy-type spaces, Dokl. Akad. Nauk 328(3) (1993), 281-284. (Russian)

Institute of Mathematics and Mechanics of NAS of Azerbaijan, Baku, AzERBAIJAN

E-mail address: b_bilalov@mail.ru

Ganja State University, Ganja, Azerbaijan

E-mail address: aysel_guliyeva20@mail.ru

Institute of Mathematics and Mechanics of NAS of Azerbaijan, Baku, Azerbaijan; Khazar University, Baku, Azerbaijan

E-mail address: s_sadigova@mail.ru 